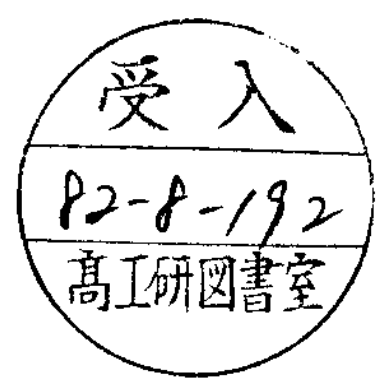

EUROPEAN ORGANIZATION FOR NUCLEAR RESEARCH

CERN-EP/82-89

29 June 1982

\title{
LARGE TRANSVERSE MOMENTUM PARTICLE PRODUCTION
}

\section{IN $\alpha \alpha$ AND PP COLLISIONS AT THE CERN ISR}

The Axial Field Spectrometer Collaboration

$\left(\right.$ Brookhaven $^{1}-$ CERN $^{2}-$ Copenhagen $^{3}-$ Lund $^{4}-$ Pennsylvania $^{5}-$ RAL $^{6}-$ Tel-Aviv $\left.^{7}\right)$

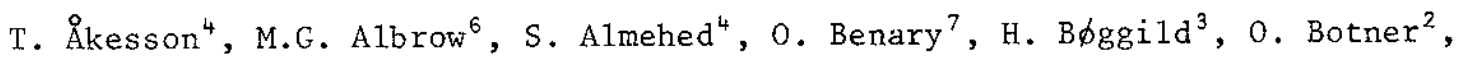
H. Brody ${ }^{5}$, V. Burkert ${ }^{2}$, A. Di Ciaccio ${ }^{1}$, D. Cockerill ${ }^{6}$, S. Dagan $^{7}$, E. Dahl-Jensen ${ }^{3}$, I. Dah1-Jensen ${ }^{3}$, G. Damgaard ${ }^{3}$, W.M. Evans ${ }^{6}$, C.W. Fabjan ${ }^{2}$,

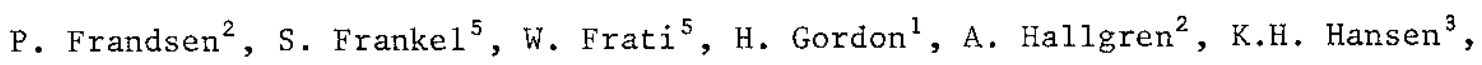
B. Heck ${ }^{2}$, H.J. Hilke ${ }^{2}$ J.E. Hooper ${ }^{3}$, G. Jarlskog ${ }^{4}$, P. Jeffreys ${ }^{6}$, G. Kesseler ${ }^{2}$, T. Killian', J. v.d. Lans ${ }^{2}$, D. Lissauer ${ }^{7}$, B. Lörstad ${ }^{4}$, T. Ludlam ${ }^{1}$, N.A. McCubbin ${ }^{6}$, A. Melin ${ }^{4}$ U. Mjornmark ${ }^{4}$,

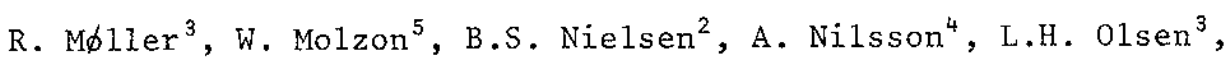

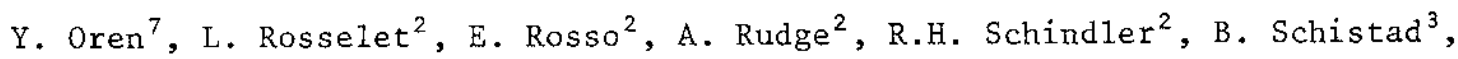
W.J. Willis ${ }^{2}$, M. Winik ${ }^{1}$, W. Witzeling ${ }^{2}$, M. Wood ${ }^{5}$, C. Woody ${ }^{1}$.

\section{$\underline{\text { ABSTRACT }}$}

The production of charged hadrons with high $\mathrm{p}_{\mathrm{T}}$ in $\alpha \alpha$ collisions at $\sqrt{\mathrm{s}}=126 \mathrm{GeV}$ and pp collisions at $\sqrt{\mathrm{s}}=31$ and $63 \mathrm{GeV}$ is compared, and the structure of the events associated with the high $-\mathrm{p}_{\mathrm{T}}$ particles is studied. The probability of finding associated particles close to the trigger particle increases strongly between $\sqrt{\mathrm{s}}=31$ and $63 \mathrm{GeV}$ for $\mathrm{pp}$ collisions. For $\mathrm{p}_{\mathrm{T}}>2.5 \mathrm{GeV} / \mathrm{c}$. the $\alpha \alpha / \mathrm{pp}$ cross-section ratio at the same energy per nucleon is measured to be $18.7 \pm 2.0$, to be compared with $A^{2}=16$, and a higher associated multiplicity is observed for $\alpha \alpha$.

(Submitted to Nuclear Physics B)

\footnotetext{
${ }^{1}$ Brookhaven National Laboratory, Upton, LI, NY, USA*)

${ }^{2}$ CERN, Geneva, Switzerland.

${ }^{3}$ Niels Bohr Institute, University of Copenhagen, Denmark.

${ }^{4}$ University of Lund, Sweden.

${ }^{5}$ University of Pennsylvania, USA ${ }^{*}$

${ }^{6}$ Rutherford Appleton Laboratory, Didcot, UK.

${ }^{7}$ University of Tel-Aviv, Israel.

*) Supported in part by D.O.E.
} 


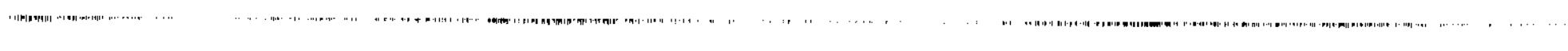


1. INTRODUCTION

The storage of $\alpha$-particle beams in the CERN Intersecting Storage Rings (ISR) in 1980 offered a unique possibility for comparing $\alpha \alpha$, $\alpha p$, and $p p$ collisions at very high energies .

It has been known since the Fermilab experiments [1] of 1975 that high- $\mathrm{p}_{\mathrm{T}}$ proton-nucleus cross-sections show anomalous features. The cross-sections vary as $\mathrm{A}^{\alpha\left(\mathrm{p}_{\mathrm{T}}\right)}$, with $\alpha\left(\mathrm{p}_{\mathrm{T}}\right)<1.0$ at low $\mathrm{p}_{\mathrm{T}}$ but rising above 1.0 at high $\mathrm{p}_{\mathrm{T}}$. Possibly these nuclear effects can be used to elucidate the mechanisms of high-p ${ }_{T}$ produc- $^{-}$ tion in hadron-hadron interactions. The ISR data provide the first opportunity to study these effects in nucleus-nucleus interactions, to study the effect at higher energies, and to study the event structure.

In this paper we shall focus on events in which a high-p $\mathrm{p}_{\mathrm{T}}$ charged particle is emitted, and compare such events in three data samples: $\alpha \alpha$ collisions at $\sqrt{\mathrm{s}}=$ $=126 \mathrm{GeV}$, and $\mathrm{pp}$ collisions at $\sqrt{\mathrm{s}}=31$ and $63 \mathrm{GeV}$. The comparison will thus comprise pp collisions with protons of the same energy as the energy per nucleon in the $\alpha \alpha$ collisions, and pp collisions at twice that energy.

\section{THE APPARATUS AND DATA-TAKING}

The experiment was performed at the CERN ISR with the Axial Field Spectrometer $[2,3]$ (fig. 1). Events with a charged high $-\mathrm{p}_{\mathrm{T}}$ particle inside the rapidity range $|y|<0.8$ were selected with a trigger system based on the inner scintillator hodoscope (IH), the two external proportional wire chambers PC1 and PC2, and information from the central drift chamber.

The trigger decision consisted of three stages. A fast pretrigger required a threefold coincidence between one of the IH counters (strips with $\Delta \phi=8^{\circ}$ ) and a hit in groups of (horizontal) wires in PC1 and PC2, with all three elements aligned with the interaction diamond. A second stage, based on a programable "RAM" matrix, aborted the event if the individual hit wires in PCl and PC2 were not aligned with the diamond. A single particle must have $\mathrm{p}_{\mathrm{T}} \geq 2 \mathrm{GeV} / \mathrm{c}$ to satisfy these conditions in the transverse magnetic field of $5 \mathrm{kG}$. However, at this stage 
the trigger condition can also be satisfied by $\geq 2$ particles giving, by chance, aligned hits in $\mathrm{IH}, \mathrm{PC} 1$, and $\mathrm{PC} 2$. Most of the events in the latter class were rejected in the third stage by requiring a track in the central drift chamber with sagitta (in the transverse view) less than a preset limit, in a small azimuthal region in line with the proportional chamber coincidence. This stage used an online microprocessor, ESOP. A more detailed description can be found elsewhere [2]. A set of Cerenkov counters in the aperture of the trigger region $\left(\Delta \phi \sim 45^{\circ}\right)$ allowed identification of $\sim 30 \%$ of the high- $\mathrm{p}_{\mathrm{T}}$ trigger particles. However, in the data presented here we shall only comment briefly on the particle composition.

With the trigger threshold set to $\mathrm{P}_{\mathrm{T}}>2.5 \mathrm{GeV} / \mathrm{c}$ we obtain, after applying off-1ine requirements on track quality in the drift chamber, an acceptance which exceeds $50 \%$ for $\left(\mathrm{P}_{\mathrm{T}}\right)_{\text {trig }}>2.5 \mathrm{GeV} / \mathrm{c},|\mathrm{y}|<0.6$, and azimuthal angle $|\phi|<20^{\circ}$. Consistency was required between the extrapolation of the reconstructed track in the drift chamber and a matching wire hit in both PC1 and PC2. The normalization of the spectra is based on a calculation of the trigger acceptance and on measurements of the luminosity using scintillation counters surrounding the downstream beam pipes. The Van der Meer method of vertical beam steering was used for calibrating these luminosity monitoring counters.

The trigger acceptance was calculated using a Monte Carlo program, taking into account the position and shape of the interaction region (the diamond), the geometry of the IH counters, and the PC1 and PC2 groups of wires which enter into the first level of the trigger system. Multiple scattering and the effects of the ESOP trigger condition were also included in the calculation of the geometrical trigger acceptance. In addition, corrections were made for detector efficiencies. The efficiency of the IH scintillators was measured to be only $\sim 75 \%$ in the $\alpha \alpha$ run and in the preceding pp run at $\sqrt{\mathrm{s}}=63 \mathrm{GeV}$, whereas it was $94 \%$ in the later pp run at $\sqrt{s}=31 \mathrm{GeV}$ after the scintillator had been replaced. The proportional chamber wire efficiency, combined with the probability for survival of a particle during its penetration of the material on the way, was measured to be $95 \%$ for PC1 and $82 \%$ for PC2 by following non-trigger tracks through the detectors. 
When two tracks are close to each other in azimuth they shadow each other to some degree in the drift chamber, such that later cuts on track quality cause a loss of trigger tracks. Assuming that the number of close crossing tracks and non-crossing tracks are the same, this effect was found to 1 ose $10 \%$ of the trigger tracks. Finally, the rather strict cuts on the quality of the drift chamber tracks in terms of $\chi^{2}$ and number of space-points are estimated to reject $18 \%$ of real associated tracks in the $\alpha \alpha$ run and $8 \%$ in the other runs. These cuts were used to reject a background of fake high- $\mathrm{p}_{\mathrm{T}}$ tracks oxiginating from decays, interactions, etc.

Some of these efficiency factors depend weakly on the local density of tracks in the apparatus. For example, the efficiency of the IH counters is affected by hits by more than one particle. Such effects have been corrected for, using our observed track densities.

Unfortunately the height of the interaction diamond differed (by several millimetres) in the different data samples, changing the relative acceptance for positive and negative particles. Rather than presenting results for positive and negative particles separately, we have combined them, after checking with the Monte Carlo program that the combined acceptance then depends very little on the diamond height.

After all the corrections, we estimate that the remaining systematic errors are independent of $\mathrm{p}_{\mathrm{T}}\left(\mathrm{p}_{\mathrm{T}}>2.5 \mathrm{GeV}\right)$ and are $\sim 20 \%$ on the normalization of the cross-section values and $\sim 10 \%$ on the ratio between the results found in the three data samples.

Data were also taken with a "minimum bias" trigger which required a hit in the inner scintillator hodoscope IH or a coincidence between the scintillators surrounding the downstream beam pipes. This sample was analysed to obtain the single charged-particle production cross-sections for $\mathrm{p}_{\mathrm{T}}<2 \mathrm{GeV} / \mathrm{c}$. Being an independent sample, the systematic exror on the $10 \mathrm{~W}^{-} \mathrm{p}_{\mathrm{T}}$ data is independent of the systematic error on the $\mathrm{p}_{\mathrm{T}}>2 \mathrm{GeV} / \mathrm{c}$ sample, and is estimated to be $\pm 15 \%$ on the $\alpha \alpha: p p$ ratio. 


\section{RESULTS}

The $\mathrm{p}_{\mathrm{T}}$ spectra obtained for the trigger particles from pp collisions at $\sqrt{\mathrm{s}}=31$ and $63 \mathrm{GeV}$ and from $\alpha \alpha$ collisions at $\sqrt{\mathrm{s}}=126 \mathrm{GeV}$ are shown in fig. 2 . It is seen that our pp measurements are quite consistent with a parametrization of previously published charged-particle spectra at y $\sim 0[4,5]$, combined at $\mathrm{p}_{\mathrm{T}}>$ $>4.5 \mathrm{GeV} / \mathrm{c}$ with spectra extracted from $\pi^{0}$ measurements [6] assuming the ratio $\pi \%$ all charged to be 0.3 .

The rapidity distributions of the trigger particle with $\mathrm{p}_{\mathrm{T}}^{\mathrm{cm}}>2.5 \mathrm{GeV} / \mathrm{c}$ in the three data samples (fig. 3) show very little variation out to $y=0.6$. This is in disagreement with two high $-\mathrm{p}_{\mathrm{T}}$ data points at $\mathrm{y}=0.6$ for $\mathrm{pp}, \sqrt{\mathrm{s}}=31 \mathrm{GeV}$, from the British-Scandinavian (BS) Collaboration [4]. Those two data points are combined and plotted in fig. 3 relative to the level at $y=0$. The rapidity dependence of the BS measurements at $\sqrt{\mathrm{s}}=53 \mathrm{GeV}$, which are more precise and abundant (also plotted in fig. 3), is in agreement with our present results, and we conclude that the two points of ref. 4 for $\sqrt{\mathrm{s}}=31 \mathrm{GeV}, \mathrm{y}=0.6$, and $\mathrm{p}_{\mathrm{T}}^{\mathrm{cm}}=2.6$ and $2.7 \mathrm{GeV} / \mathrm{c}$ are incorrect.

In $\mathrm{fig} .4$ is shown the ratio $\mathrm{R}$ of the invariant cross-sections from $\alpha \alpha$ collisions at $\sqrt{\mathrm{S}}=126 \mathrm{GeV}$ and $\mathrm{pp}$ collisions at $\sqrt{\mathrm{s}}=31 \mathrm{GeV}$, i.e. at the same c.m. energy per nucleon. The values for this ratio at $\mathrm{p}_{\mathrm{T}}^{\mathrm{cm}}<2 \mathrm{GeV} / \mathrm{c}$ are obtained from the minimum bias samples. The $\sqrt{\mathrm{s}}=31 \mathrm{GeV}$ spectrum for $\mathrm{p}_{\mathrm{T}}<2 \mathrm{GeV} / \mathrm{c}$ has been derived from a $\sqrt{\mathrm{s}}=63 \mathrm{GeV}$ spectrum with more statistics and scaled to $\sqrt{\mathrm{s}}=31 \mathrm{GeV}$ using the previously measured energy dependence versus $\mathrm{P}_{\mathrm{T}}[4,7]$. This procedure was adopted because we did not obtain good 'minimum bias' data at $\sqrt{\mathrm{s}}=31$ GeV with the apparatus in a condition identical to that in the od data run.

The ratio increases with $\mathrm{p}_{\mathrm{T}}$ in the $l o w-\mathrm{p}_{\mathrm{T}}$ region up to $\approx 2.5 \mathrm{GeV} / \mathrm{c}$, reaching a value of $\sim 19$ in the region around $3 \mathrm{GeV} / \mathrm{c}$. For $\mathrm{P}_{\mathrm{T}}^{\mathrm{cm}}>2.5 \mathrm{GeV} / \mathrm{c}$ the average ratio is $18.7 \pm 0.5$ with a systematic error of $\sim 10 \%$. This is close to the value of 16 expected in a naive model, where the $\alpha$ particles behave as four independent nucleons. A value exceeding 16 would be expected if the mechanism producing the 
"anomalous nuclear enhancement" found by the Chicago-Princeton group [1] in pnucleus collisions is also operative in $\alpha \alpha$ collisions at these higher energies. This group parametrized the A-dependence of the high-p $\mathrm{p}_{T}$ cross-section as $A^{\alpha\left(\mathrm{P}_{\mathrm{T}}\right)}$, finding $\alpha\left(\mathrm{p}_{\mathrm{T}}\right)>1$ for $\mathrm{p}_{\mathrm{T}} \gtrsim 1.5 \mathrm{GeV} / \mathrm{c}$. It is not theoretically obvious what the correct A-dependence should be for nucleus-nucleus collisions. As we have only measured $\alpha \alpha$ and pp collisions, rather than an A-dependence with different nuclei, a comparison of the data of fig. 4 with the Chicago-Princeton results can only be made in the framework of a theoretical model.

Our result is in good agreement with the ratio, found by experiment $\mathrm{R} 418$ at the CERN Split-Field Magnet [8], between the charged-particle production spectra in $\alpha \alpha$ collisions, measured in that experiment, and the pp spectra measured by the BS Collaboration [4], when the BS measurements at $y=0$ are used.

Experiment $R 806$ [9] has measured $\pi^{0}$ production in $\alpha \alpha$ and $p p$ collisions in approximately the same $p_{T}$ region as that covered by the present data. Although there is no a priori reason why the $\alpha \alpha / \mathrm{pp}$ ratios should be identical for $\pi^{\circ}$ and for charged particles, ratios extracted from the results of ref. 9 are compatible with our results, given the quoted systematic errors for the two experiments (apart from their lowest $\mathrm{p}_{\mathrm{T}}$ point at $\mathrm{p}_{\mathrm{T}} \approx 2.1 \mathrm{GeV} / \mathrm{c}$, which has a significantly lower value of R).

We have checked the possibility of a different particle composition using the Cerenkov counter identification. For both positive and negative high $-\mathrm{p}_{\mathrm{T}}$ particles we find the same relative content of heavy particles $\left(h^{+}=K^{+}, p\right.$ and $\left.h^{-}=K^{-}, \vec{p}\right)$ in the $\alpha \alpha$ data as in the pp data at $\sqrt{\mathrm{s}}=31 \mathrm{GeV}$. In the range $2<\mathrm{p}_{\mathrm{T}}<4 \mathrm{GeV} / \mathrm{c}$ we measure $\mathrm{h}^{+} / \mathrm{all}^{+}=0.49 \pm 0.04$ in $\alpha \alpha$ and $0.47 \pm 0.04$ in $\mathrm{pp}$, and $\mathrm{h}^{-} / \mathrm{all} \mathrm{l}^{-}=0.26 \pm 0.03$ in $\alpha \alpha$ and $0.26 \pm 0.03$ in $\mathrm{pp}$.

\section{CORRELATIONS WLTH OTHER PARTICLES}

We now turn to a comparison of the event structures associated with the high$\mathrm{P}_{\mathrm{T}}$ particles. 
The parameters $\mathrm{x}_{\mathrm{E}}$ and $\mathrm{p}_{\text {out }}$ are defined in the usual way, i.e. $\mathrm{x}_{\mathrm{E}}=$ $=\left|\left(\overrightarrow{\mathrm{p}}_{\mathrm{T}}\right)_{\text {trig }} \cdot\left(\overrightarrow{\mathrm{p}}_{\mathrm{T}}\right)_{\text {associated }}\right| /\left(\mathrm{p}_{\mathrm{T}}\right)_{\text {trig }}^{2}$, and $\mathrm{p}_{\text {out }}$ is the absolute value of the momentum component transverse to the plane defined by the trigger particle and the two incoming beam particles in the c.m. frame.

Figure 5 shows the $x_{E}$ distribution of associated particles on the away side with $|\mathrm{y}|<0.8$ and with $\mathrm{p}_{\text {out }}<0.5 \mathrm{GeV} / \mathrm{c}$, for events with a high-p $\mathrm{T}$ particle, $\mathrm{p}_{\mathrm{T}}>2.2 \mathrm{GeV} / \mathrm{c}$. The figure shows a close agreement for $\mathrm{x}_{\mathrm{E}}>0.3$ between the three data samples and with a result from the British-French-Scandinavian Collaboration $(R 413)$ at $\sqrt{s}=53 \mathrm{GeV}[10]$. At low $\mathrm{x}_{\mathrm{E}}(\underset{\sim}{<0.3)}$ the distribution for $\alpha \alpha$ collisions is $\sim 30 \%$ higher than for pp collisions, showing a higher multiplicity of 1 low- $\mathrm{p}_{\mathrm{T}}$ particles in the $\alpha \alpha$ data.

Figure 6 shows the distributions of the azimuthal angle relative to the trigger, $|\Delta \phi|=\left|\phi-\phi_{\text {trig }}\right|$, in three intervals of $\mathrm{p}_{\mathrm{T}}$ for the associated particles. It is seen that the $\alpha$ data look similar in shape but are at a higher level than the pp data at $\sqrt{\mathrm{s}}=31 \mathrm{GeV}$ (i.e. at the same c.m. energy per nucleon), whereas a comparison between the pp data at $\sqrt{\mathrm{s}}=31$ and $63 \mathrm{GeV}$ shows a clear increase in the number of particles close to the trigger particle when the energy is increased.

Figure 7 illustrates these observations in more detail. Here the distribution at $\sqrt{\mathrm{s}}=31 \mathrm{GeV}$ has been subtracted from the $\alpha \alpha$ distribution and from the $\sqrt{\mathrm{s}}=63 \mathrm{GeV}$ distribution, so the figure shows the distribution of the extra particles obtained by either changing from pp to $\alpha \alpha$ collisions at the same energy per nucleon or by increasing the energy in pp. It is clearly seen that the extra multiplicity in the high- $\mathrm{p}_{\mathrm{T}} \alpha \alpha$ collisions, compared to pp at $\sqrt{\mathrm{s}}=31 \mathrm{GeV}$, is very uniformly distributed in azimuthal angle. However, for pp at $\sqrt{\mathrm{s}}=63 \mathrm{GeV}$ compared to $31 \mathrm{GeV}$, the increase occurs mainly close to the trigger particle in azimuth. The corresponding increase in average momentum accompanying the trigger particle is $2250 \mathrm{MeV} / \mathrm{c}$.

Finally, in ordex to examine more closely the distribution of extra particles in $\alpha \alpha$ collisions, fig. 8 shows the difference between $p_{x}$ spectra in the $\alpha \alpha$ data 
and $\mathrm{pp}$ at $\sqrt{\mathrm{s}}=31 \mathrm{GeV}$. Here $\mathrm{p}_{\mathrm{x}}$ is the momentum in the transverse plane projected on the trigger track. It is seen that the difference spectra look alike on the trigger side and the away side. Also the distributions agree with the inclusive distribution from pp at $\sqrt{\mathrm{s}}=31 \mathrm{GeV}$ (where the transverse momenta are projected on a random axis in the transverse plane) when this is multiplied by 1.4 (dashed line). (The error on the multiplication factor is essentially systematic and is $\sim 0.2$.) Hence the additional particles in the $\alpha \alpha$ collisions do not appear to take part in the characteristic event pattern seen in high $-\mathrm{p}_{\mathrm{T}}$ PP collisions, but are distributed roughly as in general inelastic collisions.

\section{DISCUSSTON AND CONCLUSIONS}

We have measured the $\mathrm{p}_{\mathrm{T}}$ spectra of charged particles in $\alpha \alpha$ collisions at $\sqrt{\mathrm{s}}=126 \mathrm{GeV}$ and made a comparison with pp collisions at the same c.m. energy per nucleon. We find a ratio between the production cross-sections somewhat above the naive value 16 ; for $\mathrm{p}_{\mathrm{T}}>2.5 \mathrm{GeV} / \mathrm{c}, \mathrm{R}=18.7 \pm 2.0$. The associated event structure in an $\alpha \alpha$ collision producing a $\mathrm{p}_{\mathrm{T}}>2.5 \mathrm{GeV} / \mathrm{c}$ particle appears to be very much the same as that in a pp collision at the same nucleon-nucleon c.m. energy superimposed on an uncorrelated "background" with spectra of the same shape as in normal inelastic events but multiplied by 1.4. We have carried out [11] an independent study of $\alpha \alpha$ minimum bias collisions, where the mean central multiplicity is also higher than in pp collisions at the same nucleon-nucleon collision energy $\sqrt{\mathrm{s}}$. However, the increase in multiplicity associated with low- $_{\mathrm{T}}$ particles corresponds to a multiplication factor of $0.9 \pm 0.2$ instead of $1.4 \pm 0.2$ as seen in high- $\mathrm{p}_{\mathrm{T}}$ events. This difference, and the observation of the cross-section ratio exceeding 16, suggest that a soft multiple scattering mechanism enhances high-p $\mathrm{p}_{\mathrm{T}}$ particle production in $\alpha \alpha$ collisions.

Our comparison between pp collisions at $\sqrt{\mathrm{s}}=31$ and $63 \mathrm{GeV}$ shows that the away-side $x_{E}$ distribution looks approximately the same, but there is an increase in the number of high- $\mathrm{p}_{\mathrm{T}}$ particles on the trigger side. This corresponds to an average increase in accompanying momentum of $\sim 250 \mathrm{MeV} / \mathrm{c}$. Thus not only does the 
cross-section for producing a high- $\mathrm{p}_{\mathrm{T}}$ particle rise strongly between $\sqrt{\mathrm{s}}=31 \mathrm{GeV}$ and $\sqrt{s}=63 \mathrm{GeV}$ (see fig. 2), but in addition the average $\mathrm{p}_{\mathrm{T}}$ of the particles close to the trigger increases. Studies of high- $\mathrm{p}_{\mathrm{T}}$ jet structure will therefore benefit considerably from such an increase in collision energy.

\section{Acknowledgements}

We thank the CERN PS and ISR Divisions for providing beams of $\alpha$ particles in the ISR, and the EP, EF and ISR Divisions for work on the construction and installation of the Axial Field Spectrometer. We acknowledge the work of J. Lindsay, S. Delavallade, M. Kreisler and S. Jaroslawski on the trigger electronics, and the Physics Apparatus Group at RAL on the multiwire proportional chamber construction. We thank S. Bapst, M. Mazerand and M.-I. Sundell for their help. The experiment is supported by the Science Research Councils of Denmark, Sweden, and the UK, and the Dept. of Energy (USA). 


\section{REFERENCES}

[1] J.w. Cronin et a1., Phys. Rev. D 11 (1975) 3105.

[2] H. Gordon et al., Nucl. Instrum. Methods 196 (1982) 303.

[3] 0. Botner et a1., Nuc1. Instrum. Methods 196 (1982) 315.

[4] B. Alper et a1., Nuc1. Phys. B100 (1975) 237.

[5] D. Antreasyan et a1., Phys. Rev. Lett. 38 (1977) 112 and 115.

[6] C. Kourkoumelis et a1., Z. Phys. C $\underline{5}$ (1980) 95.

[7] K. Guettler et al., Phys. Lett. 64B (1976) 111.

K. Guettler et a1., Nuc1. Phys. B116 (1976) 77.

[8] w. Bell et al., Phys. Lett. 112B (1982) 271.

[9] A. Karabarbounis et a1., Phys. Lett. 104B (1981) 75.

[10] M.G. Albrow et a1., Nuc1. Phys. B145 (1978) 305.

[11] Axia1 Field Spectrometer Collaboration, Multiplicity distributions in $p \alpha$ and $\alpha \alpha$ collisions in the CERN ISR, in preparation. 


\section{Figure captions}

Fig. 1 : Transverse view of the Axial Field Spectrometer. The central cylindrical drift chamber is situated in a $5 \mathrm{kG}$ field along the collision axis. The high- $\mathrm{p}_{\mathrm{T}}$ trigger for these measurements covered the Cerenkov arm on the right, with $45 \leqslant \theta \leqslant 135^{\circ}$ and $\Delta \phi \sim 45^{\circ}$.

Fig. 2 : The inclusive invariant cross-section versus $\mathrm{p}_{\mathrm{T}}$ for charged particles in $|y|<0.6$ for $\alpha \alpha$ collisions at $\sqrt{\mathrm{s}}=126 \mathrm{GeV}$ (crosses), pp collisions at $\sqrt{\mathrm{s}}=31 \mathrm{GeV}$ (triangles), and $\sqrt{\mathrm{s}}=63 \mathrm{GeV}$ (circles). The lines represent previously published results $[4,5]$.

Fig. 3 : Distribution of rapidity for $\mathrm{p}_{\mathrm{T}}>2.5 \mathrm{GeV}$. Each distribution is normalized to unit area for $|y|<0.55$. The line and the open circle represent data from the BS Collaboration [4].

Fig. 4 : The ratio $R$ of the invariant cross-sections in $\alpha \alpha$ and pp collisions at the same c.m. energy $(15 \mathrm{GeV})$ per nucleon.

Fig. 5 : The $x_{E}$ distributions on the away side for $\left(\mathrm{p}_{\mathrm{T}}\right)_{\mathrm{trig}}>2.2 \mathrm{GeV} / \mathrm{c}$, for particles within $|y|<0.8$ having $\mathrm{p}_{\text {out }}<0.5 \mathrm{GeV}$. The dashed line represents the corresponding result from $R 413$ [10].

Fig. 6 : The $|\Delta \phi|$ distributions for particles within $|y|<0.8$ for $\left(\mathrm{p}_{\mathrm{T}}\right)_{\text {trig }}>$ $>2.5 \mathrm{GeV} / \mathrm{c}$ in three intervals of $\mathrm{p}_{\mathrm{T}}$ for the associated particles.

Fig. 7 : The difference between the $|\Delta \phi|$ distributions (for particles within $|y|<0.8)$ of the $\alpha \alpha$ and pp data at $\sqrt{\mathrm{s}}=31 \mathrm{GeV}$ and of $\mathrm{pp}$ at $\sqrt{\mathrm{s}}=$

$=63$ and $31 \mathrm{GeV}$, in three momentum intervals: $\mathrm{P}_{\mathrm{T}}<0.5 \mathrm{GeV} / \mathrm{c}$ (circles), $0.5<\mathrm{p}_{\mathrm{T}}<1.0 \mathrm{GeV} / \mathrm{c}$ (triangles) and $\mathrm{p}_{\mathrm{T}}>1.0 \mathrm{GeV} / \mathrm{c}$ (squares).

Fig. 8 : The difference between the $\left(1 / \mathrm{N}_{\text {trig }}\right) /\left(\mathrm{dn}_{\mathrm{d}} / \mathrm{dp}_{\mathrm{x}}\right)$ distributions (for particles within $|y|<0.8)$ in $\alpha \alpha$ collisions and pp collisions at $\sqrt{\mathrm{s}}=31 \mathrm{GeV}$ on the away side and on the trigger side. The line represents the inclusive $\mathrm{p}_{\mathrm{x}}$ distribution from $\mathrm{pP}$ at $\sqrt{\mathrm{s}}=31 \mathrm{GeV}$ multiplied by 1.4 . 


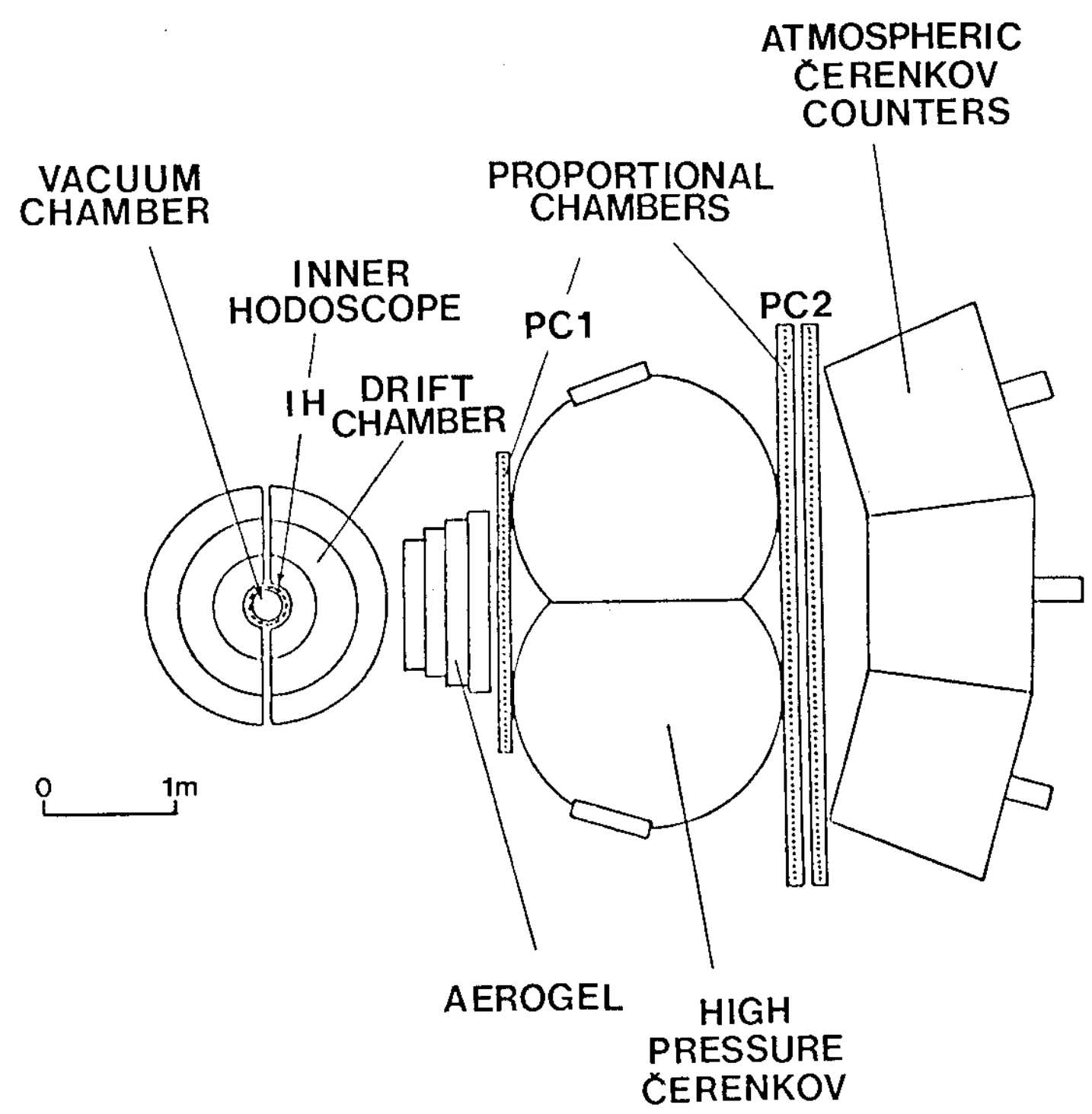

Fig. 1 


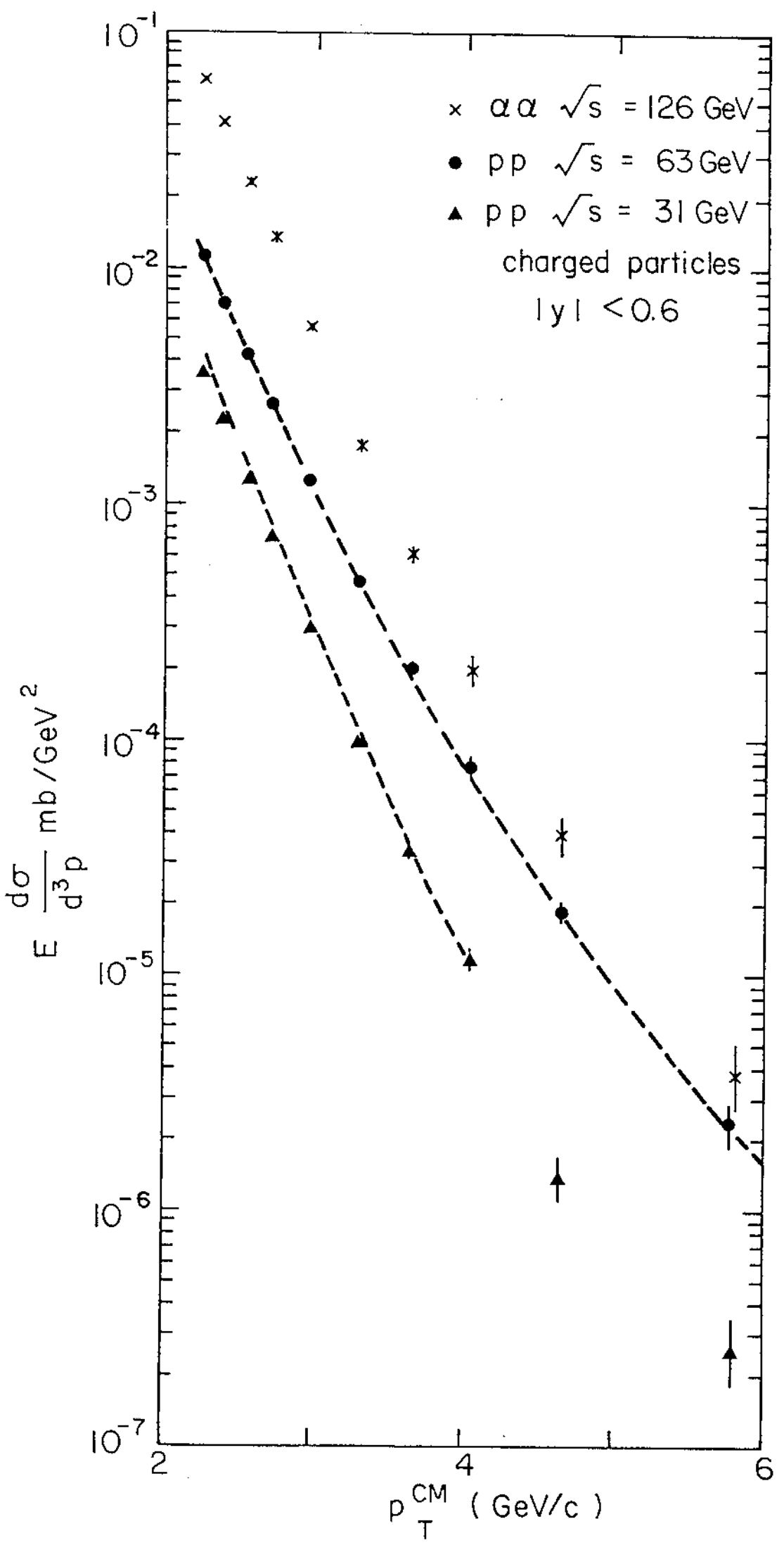

Fig. 2 


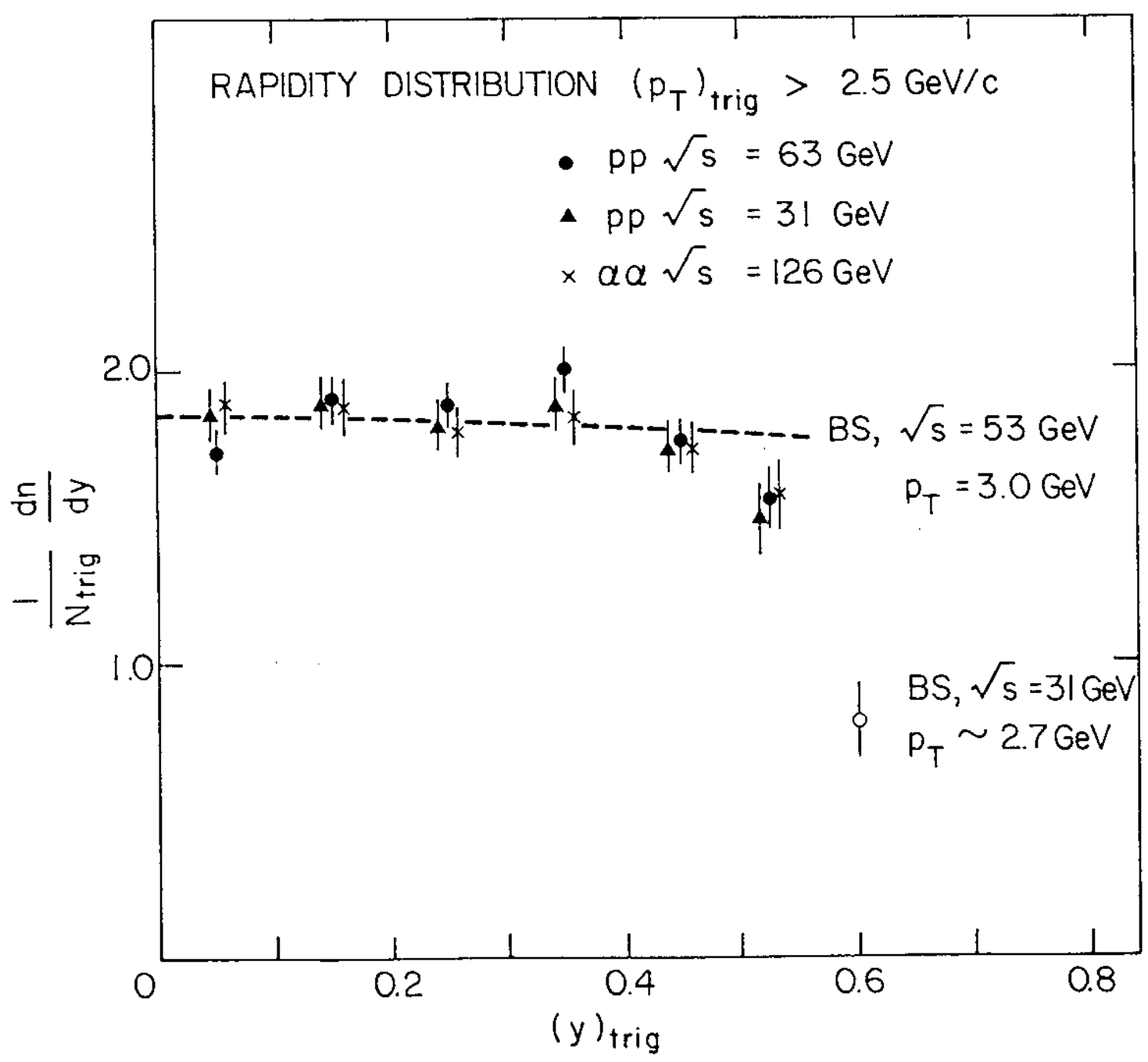

Fig. 3 


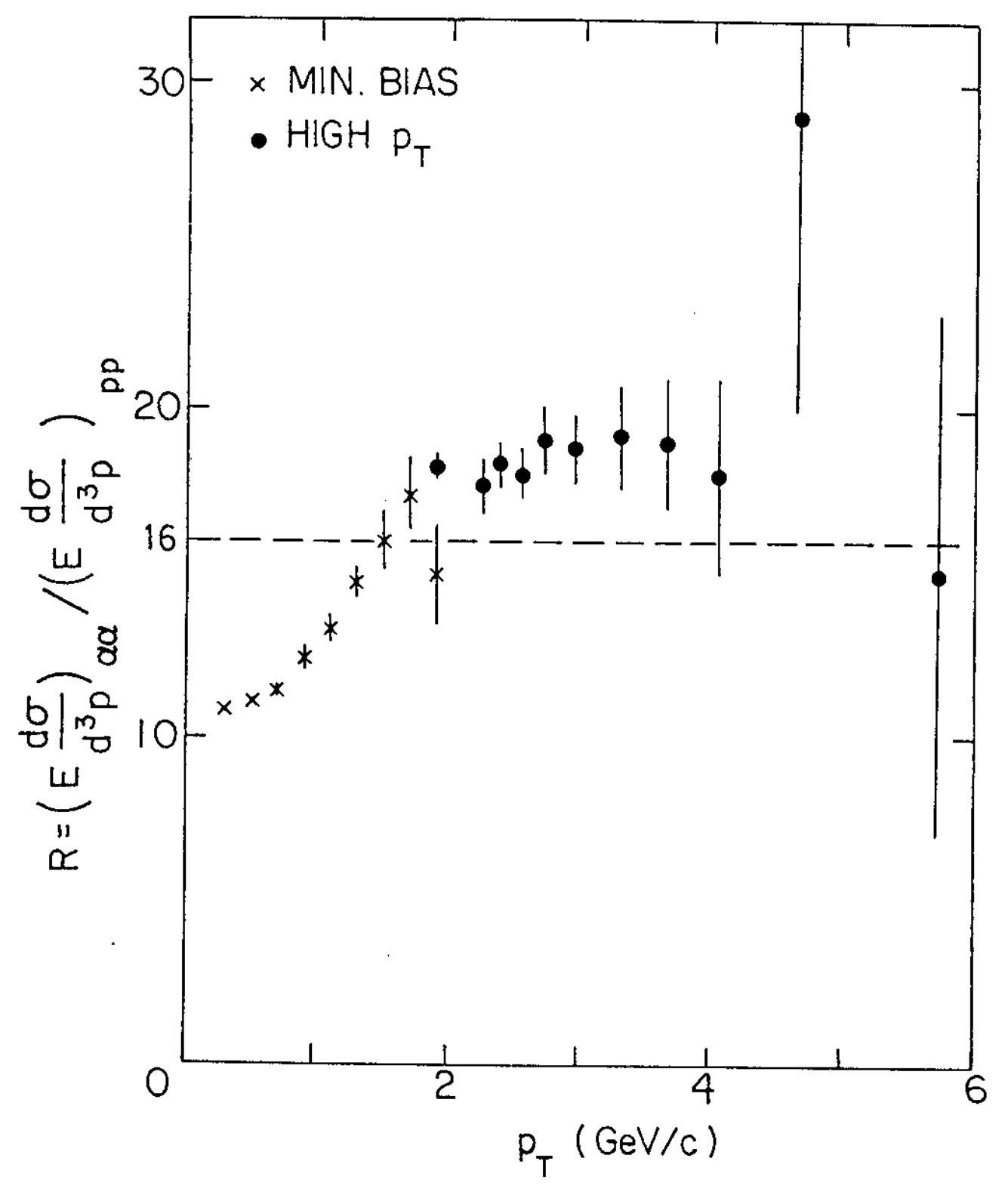

Fig. 4 


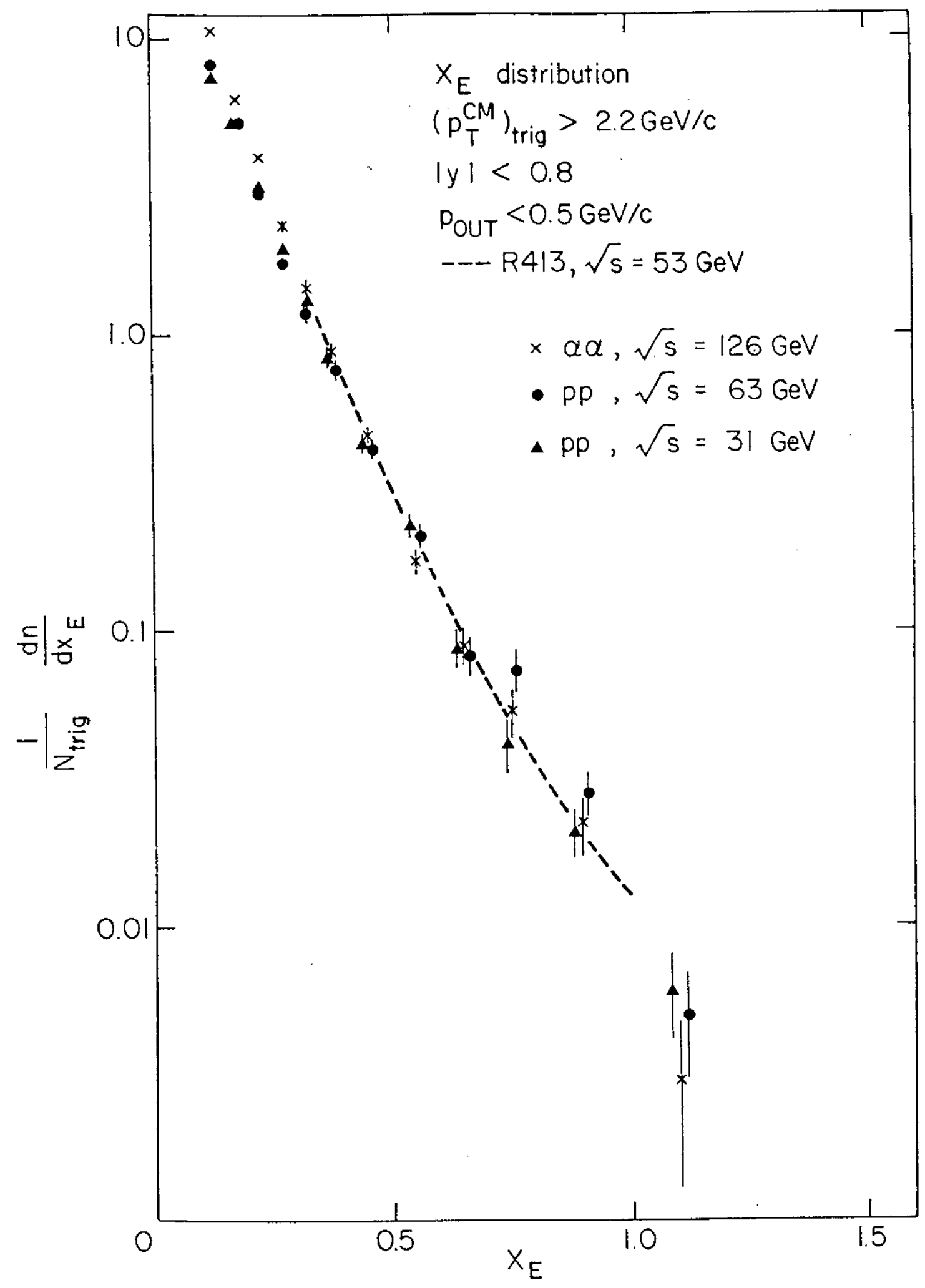

Fig. 5 
$|\Delta \Phi|$ distribution, $|y|<0.8,\left(p_{T}\right)_{\text {trig }}>2.5 \mathrm{GeV} / \mathrm{c}$
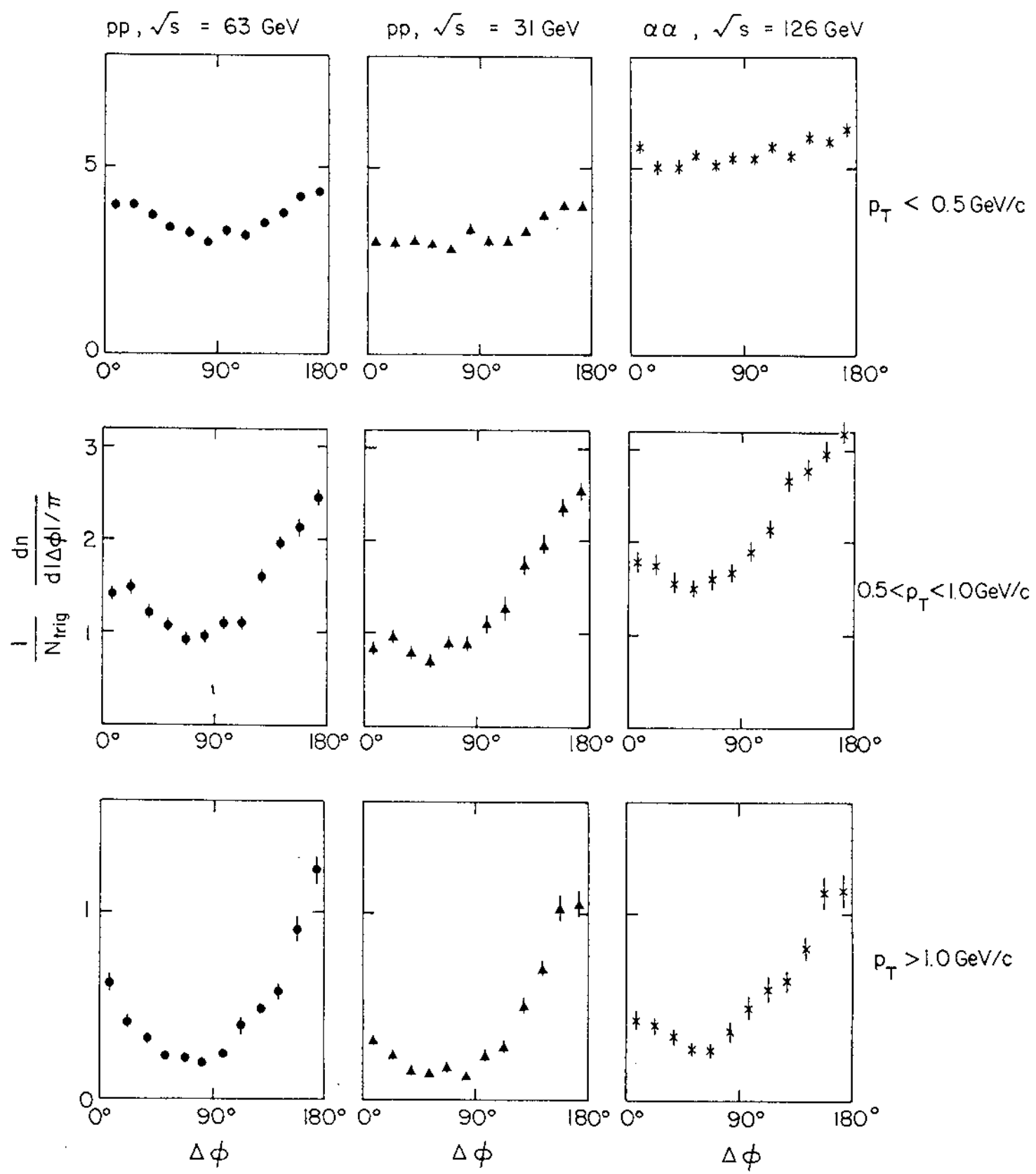

Fig. 6 

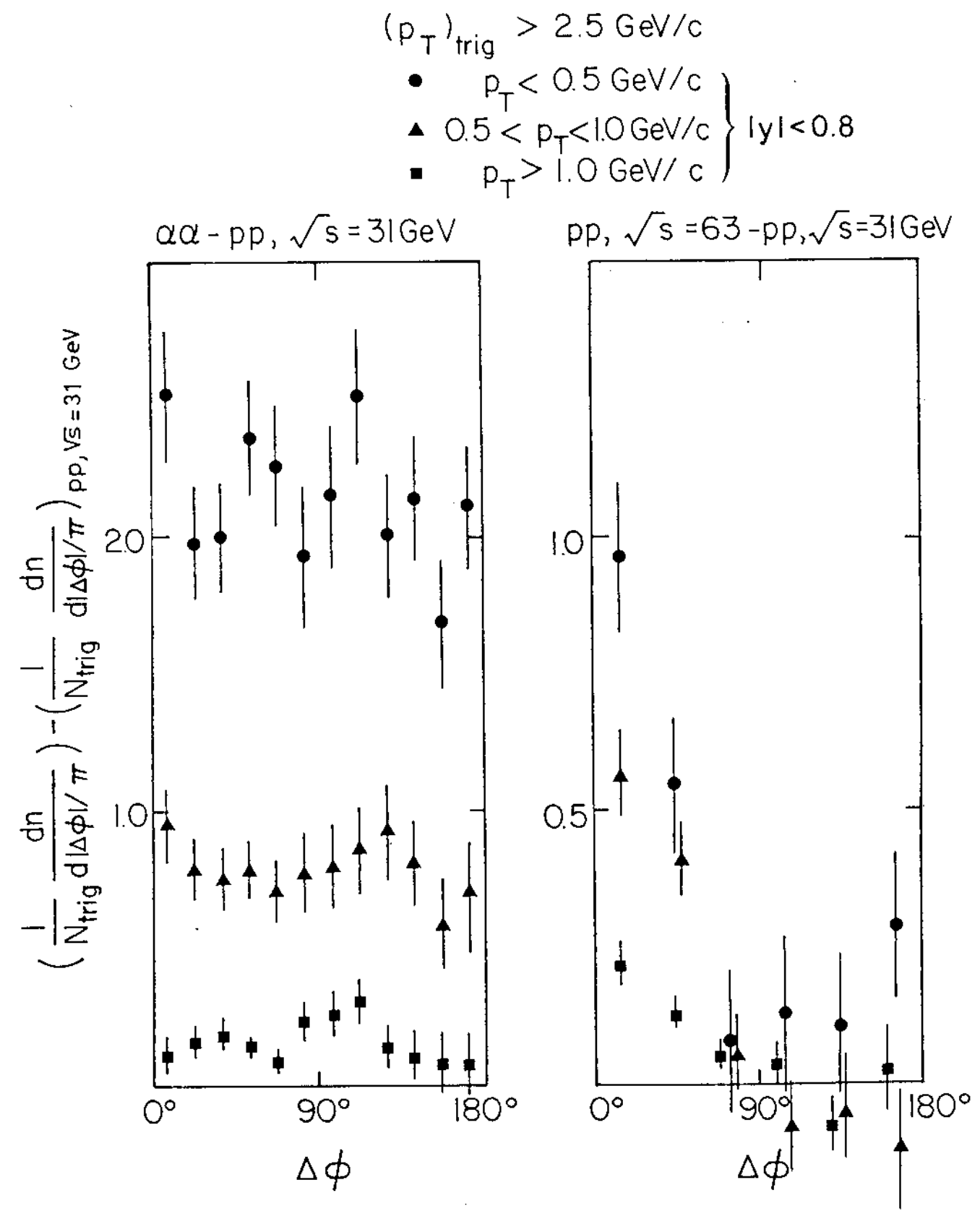

Fig. 7 


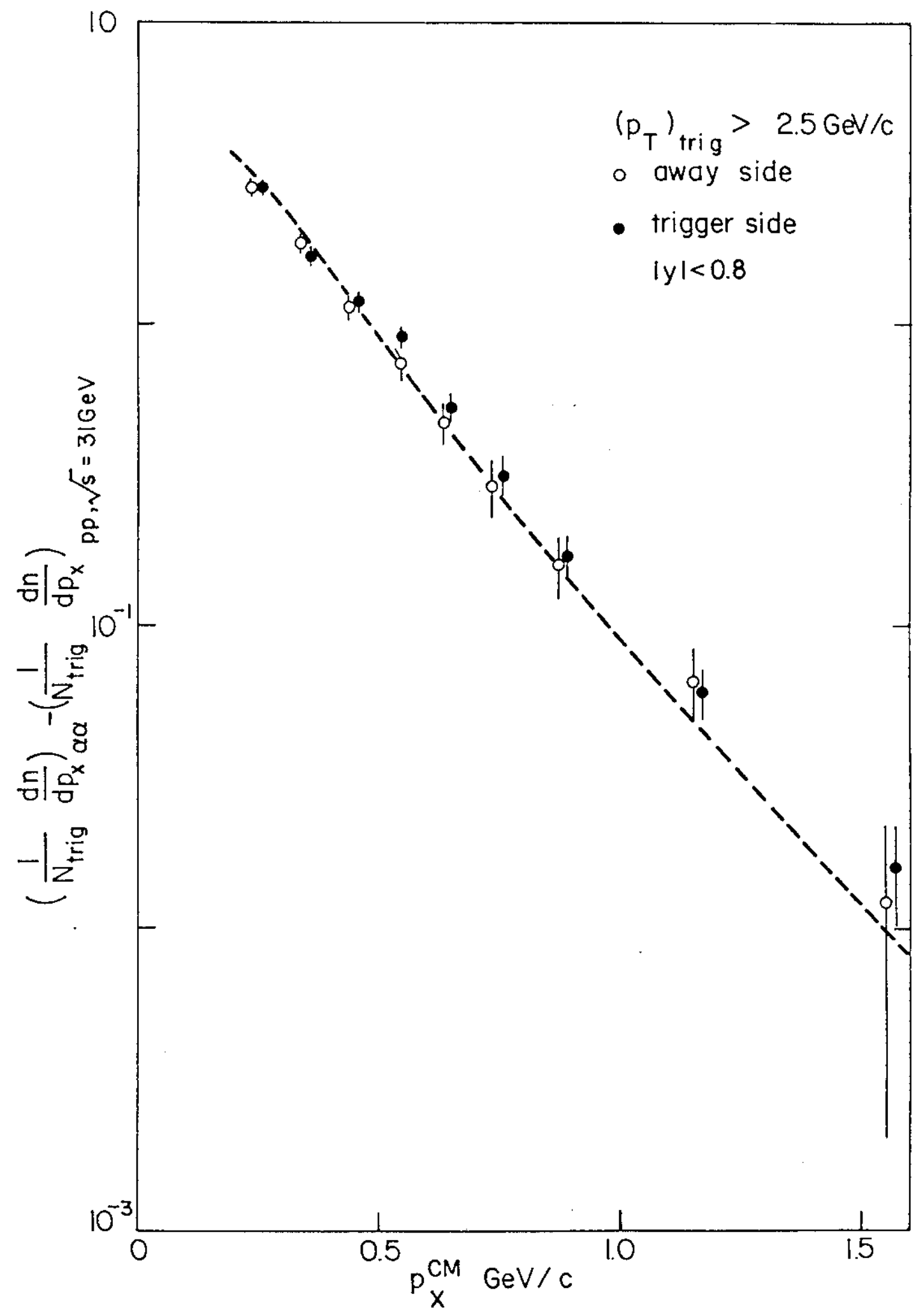

Fig. 8 